\title{
Embolización arterial supra-selectiva en otorrinolaringología: Indicaciones y complicaciones en 12 años
}

\section{Superselective arterial embolization in otorhinolaryngology: Indications and complications in a 12-year experience}

Cristián Aedo B¹, Alfredo Naser G', Pablo Cabello E², Daniel Muñoz S³, Patricio Palavicino4, Jorge Prieto4.

\begin{abstract}
RESUMEN
Introducción: Laembolización arterial es un procedimiento invasivo cuyaaplicación resulta beneficiosa en numerosas patologías otorrinolaringológicas. Se indicacomo terapia primariao como adyuvante preoperatorio principalmente en tumores vasculares de cabeza y cuello. Sus complicaciones no son infrecuentes y requieren de evaluación precoz para manejo oportuno.

Objetivo: Analizar la experiencia de nuestro servicio en el uso de embolización arterial supraselectiva en otorrinolaringología.

Material y método: Estudio retrospectivo. Se analizan los procedimientos realizados entre 1995 y 2007, se caracteriza el grupo de estudio, destacando diagnóstico, indicaciones y complicaciones del procedimiento.

Resultados: Se obtuvo un total de23 procedimientos realizados en 18 pacientes. $195,6 \%$ ( $n=22$ ) como terapia adyuvante prequirúrgica de tumores vasculares y el 4,4\% (n=1) como terapia primaria de epistaxis intratable. Se encontró la presencia de complicaciones en 21,7\% de los pacientes, con una incidencia general de 17,4\% (n=4) en complicaciones menores $y$ $4,3 \%(n=1)$ en complicaciones mayores.

Conclusiones: Es indiscutible su indicación preoperatoria en tumores vascularizados de cabeza y cuello por los beneficios quirúrgicos intraoperatorios. Las complicaciones son en su mayoría menores, las complicaciones mayores son infrecuentes, pero de un gran impacto para el paciente. En pacientes seleccionados, radiólogos preparados y materiales adecuados es una herramienta útil y segura.
\end{abstract}

Palabras claves: Embolización arterial selectiva, fibroangioma, epistaxis.

\begin{abstract} complications are not infrequent and require early assessment. experience in our clinical department.
\end{abstract}

Introduction. Arterial embolization is an invasive procedure that results beneficial in numerous otorhinolaryngological pathologies. It is mainly indicated as the primary therapy or as a presurgical adjuvant in vascular head and neck tumors. Its

Aim. To analyze the otorhinolaryngology superselective arterial embolization

\footnotetext{
Médicos Cirujanos, Otorrinolaringología, Hospital Clínico Universidad de Chile.

Médico Cirujano, Hospital Clínico Universidad de Chile.

Interno de Medicina, Hospital Clínico Universidad de Chile.

Médicos Cirujanos, Radiología Intervencional, Hospital Clínico Universidad de Chile.
} 
Material and Method. This is a retrospective study. Procedures performed between 1995 and 2007 were analyzed. The group was characterized considering diagnosis, indications and complications derived from the procedure.

Results. A total of 23 procedures performed in 18 patients was studied. 95.6\% $(n=22)$ were carried out as presurgical adjuvant therapy in the treatment of vascular tumors, and $4.4 \%(n=1)$ as primary treatment for intractable epistaxis. Complications were found in $21.7 \%$ of patients, with a general incidence of $17.4 \%(n=4)$ due to minor complications and $4.3 \%(n=1)$ due to major complications.

Conclusion. Presurgical arterial embolization is recommended in highly vascular head and neck tumors because of the surgical benefits it reports. Complications are mostly minor; major complications being infrequent, but with high impact on the patient. It is thus a useful and safe tool in selected patients, when trained radiologists and adequate materials are available.

Key words: Selective arterial embolization, complication, fibroangioma, epistaxis.

\section{INTRODUCCIÓN}

La embolización arterial es un procedimiento de especial interés en el manejo de algunas patologías de cabeza y cuello ${ }^{1}$, consiste por definición en la introducción terapéutica de distintos materiales en la circulación para ocluir vasos arteriales con el fin de: controlar o evitar hemorragias, desvitalizar una estructura, tumor u órgano y reducir el flujo de sangre de una malformación arteriovenosa². Para el éxito de este procedimiento se requiere no sólo la infraestructura y equipamiento radiológico adecuado, sino además de un radiólogo intervencionista con acabados conocimientos de anatomía vascular y familiarizado con técnicas de cateterismo y punción arteria|3 ${ }^{3-5}$.

El uso de esta técnica fue descrita inicialmente hace muchos años. En 1930, Brooks la utilizó por primera vez, para el manejo de un tumor vascular de cabeza y cuello inyectando fragmentos de músculo atados a ganchos de plata en la carótida externa². En 1960 Lussenshop y Spencer inyectaron esferas de metilmetacrilato en la arteria carótida común expuesta quirúrgicamente ${ }^{3}$. Sokoloff ${ }^{4}$ fue el primero en utilizar esta técnica en 1974 para tratar dos pacientes con epistaxis incoercible mediante angiografía selectiva y embolización de la arteria maxilar interna.

A pesar de que han pasado ya varios años de las primeras descripciones de la técnica, no ha sido sino hasta hoy que ha vuelto a retomar importancia su uso en patologías de cabeza y cuello, debido a que en las últimas décadas se ha producido un gran desarrollo de esta técnica de la mano de las nuevas técnicas de estudios imagenológicos, conocimientos anatómicos vasculares y nuevos materiales de embolización y microcatéteres permitiendo la embolización de ramas de pequeño calibre de la arteria carótida externa, o sea, la embolización selectiva. De esta forma han ido disminuyendo las complicaciones que se presentaban con las técnicas iniciales ${ }^{6,7}$.

Actualmente existen diversos estudios que avalan su uso en el manejo de varias patologías de cabeza y cuello. Esta técnica encuentra indicación como terapia primaria o como tratamiento coadyuvante preoperatorio de tumores vascularizados de cabeza y cuello ${ }^{8}$ (Tabla 1 ).

Su uso prequirúrgico en resección de fibroangiomas está ampliamente apoyado en la literatura, ya que reduce el sangrado intraoperatorio, el tiempo quirúrgico y el tamaño tumora|9-13.

El material de embolización a utilizar va a depender del calibre del vaso, patología de base y técnica a utilizar. Las variantes pueden ser en cuanto a tamaño y tipo, entre los más usados se encuentran polivinilalcohol (PVA), Gelfoam, Microcoils, Avitene ${ }^{\circledR}$, Eembospheros ${ }^{\circledR}$ y otros agentes líquidos ${ }^{15,16}$.

Las complicaciones de este procedimiento no son infrecuentes. Se clasifican en mayores con una incidencia que varía según las series, llegando hasta $6 \%$ y menores con una frecuencia también variable entre $2 \%$ a $47 \%$, $14,16-19,25$. Las complicaciones menores incluyen cefalea, fiebre, algia facial entre otras y se relacionan a la necrosis de los tejidos 0 al uso del medio de contraste, estas 
Tabla 1. Indicaciones de emboloterapia arterial en patologías de cabeza y cuello

\begin{tabular}{|ll|}
\hline Terapia primaria & Tratamiento coadyuvante preoperatorio \\
\hline Epistaxis intratables & Tumores vasculares: \\
Trauma craneofacial con ruptura arterial & - Fibroangioma juvenil \\
y/o formación de pseudoaneurisma & - Hemangiomas \\
Epistaxis posterior a cirugías & - Paragangliomas \\
Fístulas arterio-venosas & - Metástasis orofaríngeas hipervascularizadas \\
& Tumores neurogénicos \\
& Malformaciones arterio-venosas \\
& Meningiomas \\
\hline
\end{tabular}

complicaciones no dejan secuelas y son transitorias. Las complicaciones mayores en cambio, llevan a prolongar los días de hospitalización con secuelas permanentes 0 incluso la muerte, entre ellas tenemos: accidentes cerebrovasculares, ceguera, parálisis del nervio facial y necrosis de tejidos blandos entre otras (Tabla 2).

El propósito de nuestro trabajo es presentar la experiencia del uso de la embolización arterial supraselectiva en el Servicio de Otorrinolaringología del Hospital Clínico de la Universidad de Chile en los últimos 12 años. El objetivo de nuestro trabajo es presentar las indicaciones y las complicaciones en su uso.

\section{MATERIAL Y MÉTODO}

Se realizó un estudio retrospectivo de todos los pacientes a los cuales se les realizó embolización arterial supraselectiva para el manejo de patologías otorrinolaringológicas entre marzo de 1995 y di- ciembre de 2007 en el Hospital Clínico de la Universidad de Chile. Se estudiaron 18 pacientes a los cuales se les realizó el procedimiento en el Servicio de Radiología de nuestro hospital. Se analizaron los protocolos de 23 embolizaciones realizadas a estos pacientes.

A todos los pacientes se les realizó inicialmente una angiografía diagnóstica y luego la embolización supraselectiva en la misma sesión.

A todos los pacientes se les realizó una punción y cateterización transfemoral con catéter guía hasta la arteria carótida externa y luego con microcatéter de forma supraselectiva.

Se utilizaron coils de $3 \mathrm{~mm}$ para el manejo de epistaxis y PVA (50-500 $\mu$ ) y Gelfoam para manejo preoperatorio de tumores vasculares.

\section{RESULTADOS}

En el período que comprendió el estudio (19952007) se realizaron 23 procedimientos de

Tabla 2. Complicaciones de la embolización arterial selectiva de ramas de la arteria carótida externa

\begin{tabular}{|ll|}
\hline Complicaciones menores & Complicaciones mayores \\
\hline Algia facial & Accidente cerebrovascular \\
Cefalea & Ceguera \\
Fiebre & Hemiplejia \\
Parestesias & Oftalmoplejia \\
Dolor mandibular & Parálisis del nervio facial \\
Dolor en el sitio de punción & Convulsiones \\
Hematoma inguinal & Necrosis de tejidos blandos \\
Edema facial & Sepsis \\
& Muerte \\
\hline
\end{tabular}


embolización arterial supraselectiva, sobre un total de 18 pacientes. Sus indicaciones fueron: a) como tratamiento coadyuvante preoperatorio de tumores vasculares de cabeza y cuello en el $95,6 \%(n=22)$ y b) como tratamiento primario de una epistaxis de difícil manejo en el $4,4 \%(n=1)$. Los tumores vasculares embolizados fueron en su mayoría fibroangiomas (incluyendo recidivas) en el 95,5\% y un caso de hemangioma maxilar $(4,5 \%$ ) (Tabla 3). De los 16 pacientes con fibroangioma 4 presentaron recidiva (uno de estos con doble recidiva).

Del grupo en estudio, la mayoría $(80 \%$ de la muestra) se ubicó en el rango etario de entre $10 \mathrm{y}$
19 años, todos ellos con fibroangioma. Le siguen en frecuencia el grupo de entre 20 y 29 años (2 pacientes) y dos pacientes en el rango de 30 a 49 años (Figura 1). La totalidad de los pacientes correspondía al sexo masculino.

Con respecto a las complicaciones del procedimiento, se encontró una incidencia general de $21,7 \%$, de los cuales 4 casos $(17,4 \%)$ correspondieron a complicaciones menores y 1 caso $(4,3 \%)$ a una complicación mayor, en una recidiva tumoral (Figura 2).

Del análisis de estas complicaciones se desprende que dentro de las complicaciones menores,

Tabla 3. Indicaciones de embolización

\begin{tabular}{|lccc|}
\hline Indicaciones & $\begin{array}{c}\text { Pacientes } \\
\mathrm{N}^{\circ}\end{array}$ & $\mathrm{N}^{\circ}$ & $\%$ \\
\hline Tratamiento preoperatorio tumores vasculares & & & \\
$\quad$ Fibroangioma (primera resección) & 16 & 16 & 69,5 \\
$\quad$ Fibroangioma (recidiva) & $(4)$ & 5 & 21,7 \\
$\quad$ Hemangioma maxilar & 1 & 1 & 4,3 \\
$\quad$ Subtotal & 17 & 22 & 95,5 \\
Tratamiento primario & 1 & 1 & 4,5 \\
$\quad$ Epistaxis intratable & 1 & 1 & \\
$\quad$ Subtotal & 18 & & 23 \\
Total & & & \\
\hline
\end{tabular}

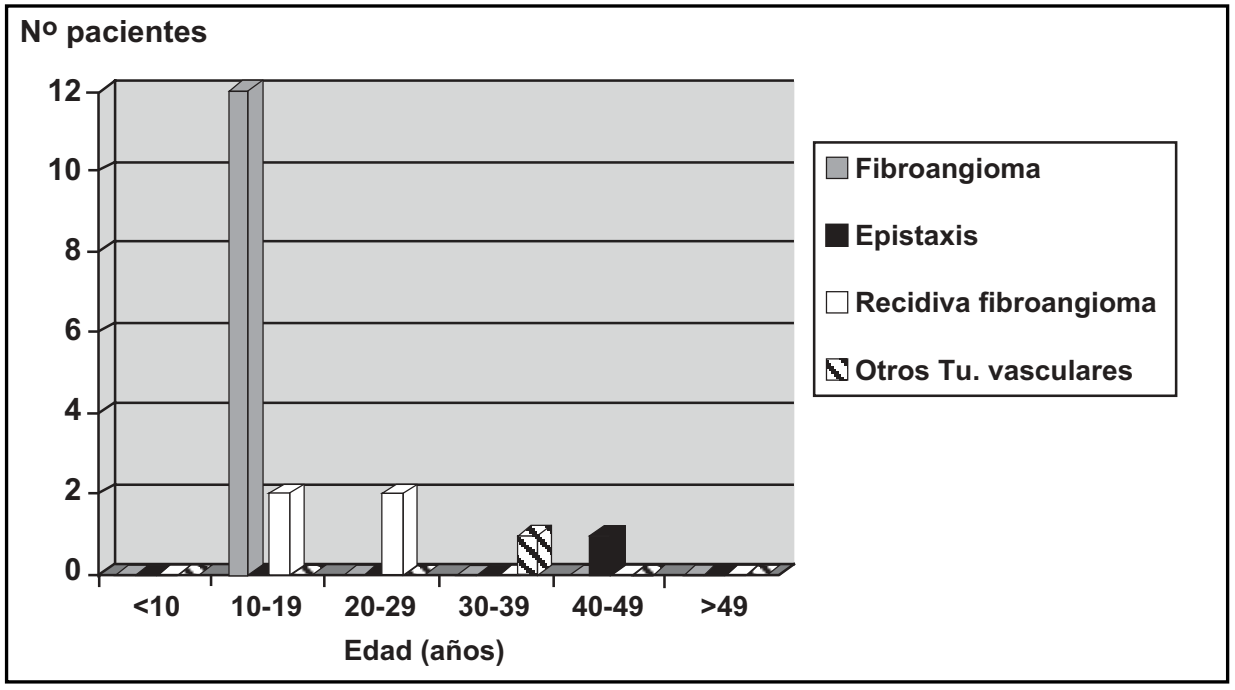

Figura 1. Rangos de edad según patología. 


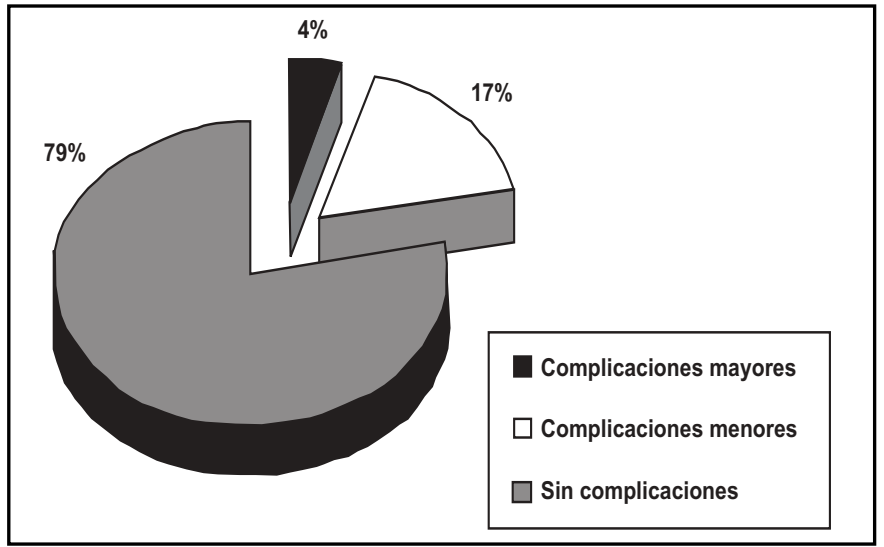

Figura 2. Incidencia de complicaciones.

la más frecuente correspondió a algia facial en $75 \%(n=3)$ y un caso de fiebre aislada post procedimiento, sin mayor repercusión (Tabla 4). En cuanto a las complicaciones mayores, se encontró un caso de amaurosis unilateral secundaria a obs-

Tabla 4. Resumen de complicaciones encontradas durante los procedimientos revisados

\begin{tabular}{|lcl|}
\hline Complicaciones & Casos & Evento \\
\hline Mayores & 1 & Amaurosis unilateral \\
Menores & 3 & Algia facial \\
& 1 & Fiebre \\
Total & 5 & \\
\hline
\end{tabular}

trucción parcial de la arteria central de la retina, en un paciente con recidiva tumoral. El cuadro fue temporal y su recuperación fue parcial con uso de corticoides. Todas las complicaciones se presentaron en el manejo de fibroangiomas no habiendo complicaciones en el manejo primario de epistaxis intratable (Tabla 5).

\section{DISCUSIÓN}

La embolización arterial supraselectiva encuentra actualmente varias indicaciones avaladas por la literatura. Su uso en el manejo de epistaxis que no responden a manejo conservador muestran tasas

Tabla 5. Complicaciones según su indicación

\begin{tabular}{|lcc|}
\hline Indicaciones & $\begin{array}{c}\text { Complicación } \\
\text { menor }\end{array}$ & $\begin{array}{c}\text { Complicación } \\
\text { mayor }\end{array}$ \\
\hline Tratamiento preoperatorio tumores vasculares & & No \\
Fibroangioma (primera resección) & 3 & 1 \\
Fibroangioma (recidiva) & 1 & No \\
$\quad$ Hemangioma maxilar & No & 1 \\
Subtotal & 4 & No \\
Tratamiento primario & No & 0 \\
$\quad$ Epistaxis Intratable & 0 & 1 \\
Subtotal & 4 & \\
Total & &
\end{tabular}


de éxito que van desde el $71 \%$ al $100 \%$ para el control de la hemorragia, de acuerdo a una reciente revisión de la literatura de Christensen y col. ${ }^{7}$ entre otros ${ }^{26,27}$ (Tabla 6).

Existen numerosas publicaciones que valoran la embolización preoperatoria de tumores vasculares de cabeza y cuello (principalmente fibroangiomas) para disminuir los tiempos quirúrgicos y la hemorragia intraoperatoria, y de esta manera permitir una mejor resección del tumor ${ }^{2,6,8-13,19-24}$. Mistry y col. ${ }^{24}$ comparó el resultado quirúrgico de 2 grupos de pacientes con fibroangioma juvenil, 19 de ellos fueron embolizados preoperatoriamente y $13 \mathrm{sin}$ embolización, encontró una disminución significativa en los tiempos operatorios y de las pérdidas sanguíneas en el grupo de pacientes embolizados.

La angiografía y embolización selectiva de las ramas de la carótida externa es considerada relativamente segura, cuando hay un "sistema arterial seguro". El número de factores que tienen un rol en determinar la aparición de complicaciones son numerosos y pueden ocurrir durante 0 después de la embolización ${ }^{18}$. Las anastomosis anormales entre ambos sistemas carotídeos es el principal factor de riesgo. Según Lasjaunias y col. ${ }^{19}$ en una revisión de 880 embolizaciones de cabeza y cuello, observó que el $80 \%$ de las complicaciones mayores eran debidas a tres factores: 1) Selección inadecuada del material embólico. 2) Reflujo del émbolo (causado por espasmo vascular, insuficiente cateterización selectiva o inyección demasiado rápida de las partículas). 3) El no reconocimiento de potenciales anastomosis peligrosas entre ambos sistemas carotídeos.

La seguridad de la embolización depende de varios aspectos prácticos como: el uso de microcatéteres que pueden llegar a finas ramas de la arteria maxilar; se debe evitar la inyección enérgica de las partículas, de esta forma se evita el vasoespasmo y el reflujo del material embólico. Por otro lado deben identificarse y evitarse aquellas ramas potencialmente peligrosas de anastomosis a

Tabla 6. Trabajos publicados de embolización para el manejo de epistaxis ${ }^{7}$

\begin{tabular}{|lccc|}
\hline Serie & $\begin{array}{c}\text { Pacientes } \\
\text { № }\end{array}$ & $\begin{array}{c}\text { Éxito } \\
(\%)\end{array}$ & $\begin{array}{c}\text { Complicaciones } \\
(\%)\end{array}$ \\
\hline Elia et al & 54 & 96 & 6 \\
Sinuloto et al & 31 & 71 & 0 \\
Roberson et al & 10 & 80 & 30 \\
Merland et al & 54 & 94 & 7 \\
VanWyck et al & 3 & 100 & 0 \\
Valdazo & 1 & 73 & NE \\
Parnes et al & 19 & 82 & 5 \\
Wehrli et al & 19 & 74 & 47 \\
Hicks et al & 7 & 100 & 0 \\
Breda et al & 1 & 81 & 0 \\
Strutz et al & 1 & 91 & 3 \\
Vitek & 30 & 97 & 2 \\
Elden et al & 97 & 90 & 0 \\
Strong et al & 16 & 88 & 8 \\
Tseng et al & 14 & 97 & 16 \\
Moreau et al & 45 & 79 & 0 \\
Cullen et al & 39 & 82 & 8 \\
Scaramuzzi et al & 12 & 89 & 0 \\
Leppanen et al & 37 & 96 & 45 \\
Klein et al & 26 & 95 & 13 \\
Oguni et al & 37 & 81 & \\
Merol et al & 16 & & \\
\hline
\end{tabular}


la arteria carótida interna como la arteria meníngea media ${ }^{26}$.

En nuestra revisión observamos que la embolización preoperatoria en tumores vascularizados de cabeza y cuello es de rutina, sin embargo su uso para el manejo de epistaxis es muy inusual $(n=1)$, prefiriéndose alternativas como la ligadura abierta 0 endoscópica. Nuestra incidencia de complicaciones concuerda con la literatura. El algia facial fue la complicación menor más frecuente, probablemente relacionada con la necrosis de tejido por avascularización, fue autolimitada y manejable con analgésicos comunes. Tuvimos una complicación mayor correspondiente a un caso de amaurosis durante la embolización, lo que sugiere obstrucción de la arteria central de la retina por material embólico, a diferencia de la neuritis óptica secundaria al medio de contraste que se presentan en diferido al procedimiento. Cabe destacar que este paciente presentaba una recidiva tumoral, en donde los mecanismos de revascularización y neoformación vascular hacen que exista un mayor riesgo de anastomosis anómalas entre ambos sistemas carotídeos. Esta complicación fue manejada con corticoides endovenosos, recuperando parcialmente la visión.

\section{CONCLUSIONES}

Como terapia coadyuvante preoperatorio es indiscutible el beneficio de la embolización arterial en tumores vascularizados de cabeza y cuello, pues disminuye el tamaño tumoral, la hemorragia intraoperatoria, facilita resección completa y, por tanto, acorta el tiempo quirúrgico. En nuestro servicio es de rutina su indicación preoperatoria en fibroangiomas primarios y en recidivas.

En epistaxis intratables no existe la experiencia mínima en nuestro servicio para poder avalar su uso en estos casos, a pesar de las tasas de éxito que muestra la literatura.

La embolización supraselectiva parece ser un procedimiento seguro con bajas incidencias de complicaciones mayores, sin embargo cuando éstas se presentan son de un gran impacto para el paciente, con secuelas permanentes y en algunos casos incluso la muerte.

\section{BIBLIOGRAFÍA}

1. Turourowski B, Zanella FE. Interventional Neuroradiology of the Head and Neck. Neuroimaging Cin N Am 2003 Aug; 13(3): 619-45.

2. BRooks B. The Treatment of Traumatic Arteriovenous Fistula. South Med J 1930; 23 : 100-6.

3. Sмiтн TP. Embolization in the External Carotid Artery. J Vasc Interv Radiol 2006 Dec; 17(12): 1897-912.

4. Sokoloff J, Wickbom I, McDonald D, Brahme F, Goergen TC, Goldberger LE. Therapeutic Percutaneous Embolization in Intractable Epistaxis. Radiology 1974 May; 111(2): 285-7.

5. Tseng E, Narducci CA, Willing SJ, Sillers MJ. Angiographic Embolization for Epistaxis: A review of 114 cases. Laryngoscope 1998; 108: 615-9.

6. Johnson MH, Chiang VL, Ross DA. Interventional Neuroradiology Adjuncts and Alternatives in Patients with Head and Neck Vascular Lesions. Neurosurg Cin NAm 2005 Jul; 16(3): 547-60.

7. Christensen NP, Smith DS, Barnwell SL, Wak MK. Arterial Embolization in the Management of Posterior Epistaxis. Otolaryngol Head Neck Surg 2005; 133: 748-53.

8. Accreditation Council on Graduate Medical Education. External Carotid Artery Embolization. American Journal of Neuroradiology September 2001; 22: S12-S13

9. De Vincentils M, Gallo A, Minni A, Torri E, Tomassi R, Della R. Preoperative Embolization in the Treatment Protocol for Rhinopharyngeal Angiofibroma: comparison of the effectiveness of various materials. Acta Otorhinolaryngol Ital 1997; 17: 225-32.

10. Gruber A, Bavinzski G, Killer M, Richling B. Preoperative Embolization of Hypervascular Skull Base Tumors. Minim Invasive Neurosurg 2000 Jun; 43(2): 62-71.

11. Roche PH, Paris J, Regis J, Moulin G, Zanaret M, Thomassin JM et al. Management of Invasive Juvenile Nasopharyngeal Angiofibromas: the role of a multimodality approach. Neurosurgery 2007 0ct; 61(4): 768-77.

12. Gupta AK, Purkayastha S, Bodhey NK, Kapilamoorthy TR, Kesavadas C. Preoperative Embolization of 
Hypervascular Head and Neck Tumours. Australasian Radiology 2007 0ct; 51(5): 446-52.

13. LI JR, QIAN J, Shan XZ ET AL. Evaluation of the Effectiveness of Preoperative Embolization in Surgery for Nasopharyngeal Angiofibroma. Eur Arch Otorhinolaryngol 1998; 255: 430-2.

14. Scaramuzzi N, Walsh RM, Brennan P, Walsh M. Treatment of Intractable Epistaxis using Arterial Embolization. Ain Oolaryngol Allied Sci 2001 Aug; 26(4): 307-9.

15. WINDFUHR JP. Indications for Interventional Arteriography in Post-tonsillectomy Hemorrhage. J Aolaryngol 2002 Feb; 31(1): 18-22.

16. GonzÁlez C, Einersen M, GutiérRez M. Embolización Arterial en Epistaxis. Rev Otorrinolaringol Or Cab Que2006; 66: 29-32.

17. Guss J, Cohen MA, Mirza N. Hard Palate Necrosis After Bilateral Internal Maxillary Artery Embolization for Epistaxis Laryngoscope 2007; 117: 1683-4.

18. Hemingway AP, Aluison DJ. Complications of Embolization: Analysis of 410 Procedures. Radiology 1988; 166: 669-72.

19. LASJAUNIAS N. Nasopharyngeal Angiofibromas: Hazards of Embolization. Radiology July 1980; 136: 119-23.

20. Kasper GC, Welling Re, Wladis AR, Cajacob DE, Grisham AD, Tomsick TA et al. A Multidisciplinary Approach to Carotid Paragangliomas. Vasc Endovascular Surg 2006 Dec-2007 Jan; 40(6): 467-74.
21. Sittel C, Gossmann A, Jungehülsing M, Zähringer M. Superselective Embolization as Palliative Treatment of Recurrent Hemorrhage in Advanced Carcinoma of the Head and Neck. Ann Otol Rhinol Laryngol 2001 Dec; 110(12): 1126-8.

22. Crespo AM, Hernández G, Barrena MR, Guelbenzu S. Head and Neck Paragangliomas: imaging diagnosis and embolization. Acta Corrinolaringol Esp 2007 Mar; 58(3): 83-93.

23. Moulin G, Chagnaud $C$, Gras R et al. Juvenile Nasopharyngeal Angiofibroma: comparison of blood loss during removal in embolized group versus nonembolized group. Cardiovasc Intervent Radiol 1995; 18: 158-61.

24. Mistry RC, QuRESHI SS, GuPTA S et al. Juvenile Nasopharyngeal Angiofibroma: a single institution study. Indian J Cancer 2005; 42: 35-9.

25. Falagas ME, Nikou SA, Siempos II. Infections Related to Coils used for Embolization of Arteries: review of the published evidence. $J$ Vasc Interv Radiol 2007 Jun; 18(6): 697-701.

26. Sinuloto tM, Leinonen AS, Karttunen AL et al. Embolization for the Treatment of Posterior Epistaxis: an analysis of 31 cases. Arch Otolaryngol Head Neck Surg 1993; 119: 837-41.

27. Elden L, Montanera W, Terbrugge K et al. Angiographic Embolization for the Treatment of Epistaxis: a review of 108 cases. Aolaryngol Head Neck Surg 1994; 111: 44-50. 